\title{
Inflation and Nonsingular Spacetimes of Cosmic Strings
}

\author{
Inyong Cho* \\ Institute of Cosmology, Department of Physics and Astronomy, \\ Tufts University, Medford, Massachusetts 02155, USA
}

(August 25, 2018)

\begin{abstract}
Inflation of cosmic gauge and global strings is investigated by numerically solving the combined Einstein and field equations. Above some critical symmetry-breaking scales, the strings undergo inflation along the radial direction as well as the axial direction at the core. The nonsingular nature of the spacetimes around supercritical gauge and global strings is discussed and contrasted to the singular static solutions that have been discussed in the literature.
\end{abstract}

PACS number(s): 98.80.Cq, 04.20.Gz

Typeset using REVTEX

*Electronic address: cho@cosmos2.phy.tufts.edu 


\section{INTRODUCTION}

Cosmic strings are linelike topological defects that may form as a result of a phase transition in the early universe. If a string is associated with a magnetic field, it is called a gauge string, otherwise it is a global string. They have attracted much attention because of their cosmological importance: deficit angle in the spacetime geometry and a candidate for the seed of structure formation in the early universe.

It was proposed that topological defects can inflate if the symmetry-breaking scale satisfies $\eta \gtrsim \eta_{c} \sim \mathcal{O}\left(m_{p}\right)$ in Refs. [2],1]. This was later verified in numerical simulations by Sakai et al. [3]. They found, in particular, that the critical value of $\eta$ for domain walls and global monopoles is $\eta_{c} \simeq 0.33 m_{p}$. Then what about cosmic strings? There is no reason why we exclude cosmic strings out of the topological inflationary category. Recently, it was numerically proved that a $(2+1)$ dimensional gauge string can inflate by de Laix et al. [4].

In this paper, we shall numerically solve the combined Einstein and field equations for a gauge and a global string in $(3+1)$ spacetime dimensions. For the gauge string, we find that the core inflates if $\eta \gtrsim 0.25 m_{p}$ with unit winding number in the critical coupling case (Bogomol'nyi limit). For the global string, $\eta \gtrsim 0.23 m_{p}$. The critical values decrease as the winding number increases. For the gauge string, the critical value also decreases slightly as the coupling of the gauge field to the scalar field becomes weaker than the self coupling of the scalar field.

The asymptotic spacetime of a gauge string is known to be conical [5]. This spacetime exhibits a deficit angle $\Delta=8 \pi G \mu$, where $\mu \sim \eta^{2}$ is the mass per unit length of the string. When the symmetry-breaking scale is sufficiently large, the deficit angle exceeds $2 \pi$ and analyses of the static solution show that the spacetime possesses a physical singularity outside the core of the string [6] 8]. However, from our numerical results we know that supermassive strings are dynamical and undergo inflation at the core. Therefore, we believe that the static treatment of supermassive strings loses its validity and that we should treat them in a time-dependent way. 
For global strings, the singularity exists regardless of the symmetry-breaking scale. Many people have tried to find a static solution of a global string and they found that there also exists a physical singularity outside the core of the string [9] [12]. What was suggested to remove this singularity is again a time-dependent treatment of the string. Gregory 13 introduced a specific metric which has an axial time dependence and showed that this spacetime is nonsingular.

In our work, we follow the evolution of supermassive gauge and global strings in a general time-dependent metric and show that no singularity develops in the spacetimes around the strings.

In the next section, we solve the Abelian Higgs model of a gauge string and discuss its inflation and spacetime geometry. Sec. III is devoted to global strings. Our conclusions are summarized in Sec. IV. In Appendix, we show the equations in detail and the numerical algorithms.

\section{GAUGE STRING}

Let us consider the Abelian Higgs model of a string with a complex scalar field $\Phi$ and a $U(1)$ gauge field $A_{\mu}$, coupled to gravity. The action is

$$
S=\int d^{4} x \sqrt{-g}\left(\frac{\mathcal{R}}{16 \pi G}+\mathcal{L}\right)
$$

where $\mathcal{R}$ is Ricci scalar and the Lagrangian $\mathcal{L}$ is

$$
\mathcal{L}=-D_{\mu} \Phi \bar{D}^{\mu} \bar{\Phi}-\frac{1}{4} F_{\mu \nu} F^{\mu \nu}-V(\Phi \bar{\Phi})
$$

Here the covariant derivative is $D_{\mu}=\nabla_{\mu}+i e A_{\mu}$, the gauge field strength is $F_{\mu \nu}=\nabla_{\mu} A_{\nu}-$ $\nabla_{\nu} A_{\mu}$, and the potential is

$$
V(\Phi \bar{\Phi})=\frac{\lambda}{4}\left(\Phi \bar{\Phi}-\eta^{2}\right)^{2}
$$

where $e$ is the coupling constant between gauge and scalar fields, $\lambda$ is the self-coupling constant of the scalar field, and $\eta$ is the symmetry-breaking scale of the scalar field. We use a general cylindrically symmetric metric 


$$
d s^{2}=-d t^{2}+B(t, r)^{2} d r^{2}+C(t, r)^{2} r^{2} d \theta^{2}+H(t, r)^{2} d z^{2}
$$

(We use $\hbar=c=1, G=1 / m_{p}^{2}$.) In these coordinates, we can use a time-dependent generalization of the usual Nielsen-Olesen anzatz for the scalar and gauge fields

$$
\begin{gathered}
\Phi=\phi(t, r) e^{i n \theta}, \\
A_{\mu}=-\frac{n}{e} \alpha(t, r) \nabla_{\mu} \theta,
\end{gathered}
$$

where $n$ is the winding number of the string. We also set the usual boundary conditions

$$
\begin{array}{ll}
\phi(t, 0)=0, & \phi(t, \infty)=\eta, \\
\alpha(t, 0)=0, & \alpha(t, \infty)=1 .
\end{array}
$$

For the above action, the Einstein's equation is

$$
G_{\mu \nu}=8 \pi G T_{\mu \nu}
$$

where the energy-momentum tensor is given by

$$
T_{\mu \nu}=D_{\mu} \Phi \bar{D}_{\nu} \bar{\Phi}+D_{\nu} \Phi \bar{D}_{\mu} \bar{\Phi}+g^{\alpha \beta} F_{\mu \alpha} F_{\nu \beta}+g_{\mu \nu} \mathcal{L}
$$

The scalar field equation is

$$
D_{\mu} D^{\mu} \Phi=\frac{\partial V(\Phi \bar{\Phi})}{\partial \bar{\Phi}}
$$

and the gauge field equation is

$$
\nabla^{\nu} F_{\mu \nu}=i e\left(\bar{\Phi} \nabla_{\mu} \Phi-\Phi \nabla_{\mu} \bar{\Phi}\right)-2 e^{2} A_{\mu} \Phi \bar{\Phi}
$$

The detailed equations are given in the Appendix.

The static solution at a large distance from the core is

$$
d s^{2}=-d T^{2}+d R^{2}+(1-4 G \mu)^{2} R^{2} d \theta^{2}+d Z^{2},
$$

where $\mu$ is the mass per unit length of the string and proportional to $\eta^{2}$. The metric (2.6) exhibits a deficit angle $\Delta=8 \pi G \mu$. As $\eta$ increases, the deficit angle also increases. When 
the deficit angle exceeds $2 \pi$, the static solution (2.6) ceases to exist and we expect that the string becomes dynamical and undergoes inflation at the core in this regime.

Let us consider the role of the winding number for inflation. The coupling between scalar and gauge fields is given by

$$
\beta \equiv\left(\frac{m_{s}}{m_{v}}\right)^{2}=\frac{\lambda}{2 e^{2}},
$$

where $m_{s}$ and $m_{v}$ are masses of scalar and gauge fields. In the limit of critical coupling (Bogomol'nyi limit), $\beta=1$, the mass per unit length is $\mu=2 \pi|n| \eta^{2}$ [14]. Then the critical value of $\eta$ at which the static solution (2.6) ceases to exist is proportional to $1 / \sqrt{n}$. Therefore, as the winding number increases, the core of the string can inflate at lower symmetrybreaking scales. Another way to explain the inflation of topological defects is the core size being bigger than the horizon scale [2,1]. Under this condition the gravitational effect becomes important in the core region and the defects are supposed to inflate. The energy density of the string can be roughly estimated by

$$
\rho \sim H^{2} \sim \frac{\mu}{\delta^{2}} \sim \frac{n \eta^{2}}{\delta^{2}},
$$

where $\delta$ is the core size and $H$ is the Hubble parameter. The core size is then

$$
\frac{\delta}{H^{-1}} \sim \sqrt{n} \eta .
$$

The core of the string can inflate when its size becomes comparable to the horizon scale, $\delta / H^{-1} \sim 1$. This condition gives again $\eta_{c} \sim 1 / \sqrt{n}$ from Eq. (2.7). In other words, for a given symmetry-breaking scale $\eta$, the core size $\delta$ is proportional to $\sqrt{n}$. Therefore, as the winding number increases, the wider core of the string can inflate at lower symmetry-breaking scales. Figure 1 shows the core sizes as functions of the winding number for several $\eta$ 's in the flat spacetime.

One important effect of the gauge field coupled to the scalar field is that it makes the string well localized and, thereby, suppresses the energy divergence along the radial direction. As the coupling constant $\beta$ increases, the effect of the gauge field becomes weaker, therefore, 
the core size becomes bigger. The string has a better chance to inflate with the bigger core acquired as $\beta$ increases.

We numerically solve the field equations introduced earlier. Initially we assume a flat spacetime and obtain the initial scalar and gauge fields, $\phi(0, r)$ and $\alpha(0, r)$, by numerically solving the flat-spacetime field equations. We then solve the time-dependent field equations to trace the evolution of these fields as well as the gravitational field $g_{\mu \nu}$. We define the core size by the proper radius $C r$ at which the scalar field is $\phi=\eta / 2$ and determine whether inflation occurs by observing the growth of this proper radius. For $n=1$ and $\beta=1$, the critical value of $\eta$ for inflation is found to be $\eta_{c} \simeq 0.25 m_{p}$. This value is remarkably close to the critical value $0.255 m_{p}$ found by Laguna and Garfinkle [8], at which the static solution becomes singular. The critical value decreases as $n$ and $\beta$ increase as expected. The critical

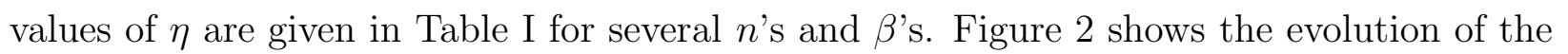
scalar field configuration as a function of the proper radius. $t$ and $r$ are scaled in the unit of the horizon size at the center, $H_{0}^{-1}=[8 \pi G V(\phi=0) / 3]^{-1 / 2}$. We can see clearly that the proper radius grows very rapidly (exponentially) in the core region.

Having found the inflationary behavior in the supermassive stringf, it is worth while to discuss its spacetime. For the supermassive string, the static solution (2.6) is no longer valid. However, since the energy-momentum tensor of the string falls off rapidly outside the core, the asymptotic form of the metric must be one of the two Levi-Civita metrics 15],

$$
d s^{2}=-d t^{2}+d z^{2}+d r^{2}+\left(a_{1} r+a_{2}\right)^{2} d \theta^{2}
$$

which is conical, or

$$
d s^{2}=\left(b_{1} R+b_{2}\right)^{4 / 3}\left(-d T^{2}+d Z^{2}\right)+d R^{2}+\left(b_{1} R+b_{2}\right)^{-2 / 3} d \theta^{2}
$$

which is a special case of a Kasner metric [16]. The qualities of the metrics (2.8) and (2.9) depend on the signs of the constants $a_{1}, a_{2}, b_{1}$, and $b_{2}$. When $a_{1}$ and $a_{2}$ have the same

\footnotetext{
1 "Supermassive" was named for the gauge string to which the static solution (2.6) no longer
} applies 
sign, the metric (2.8) is equivalent to the metric (2.6). For $b_{1}$ and $b_{2}$ with different signs, Laguna and Garfinkle [8] examined that the asymptotic spacetime of the supermassive string is described by the metric (2.9). For $a_{1}$ and $a_{2}$ with different signs, Ortiz [7] demonstrated that the metric (2.8) can also be a solution. In both cases, the spacetimes possess physical singularities at $r=-a_{2} / a_{1}$ and $R=-b_{2} / b_{1}$. The singularity approaches the core as $\eta$ increases. Nevertheless, it is not clear whether it is physically sensible to have a singularity in the string spacetime. Since we have shown that the supermassive string undergoes inflation, we believe that the static treatment is no longer valid and the string should be treated in a time-dependent way to describe its dynamical nature. We expect that the time-dependent treatment removes the singularity from the spacetime of the supermassive string.

One simple way to examine the existence of a physical singularity is to analyze the metric. The Riemann tensor contains the second derivatives of the metric terms. If the metric terms do not contain cusps and are smooth functions of the coordinates up to the second derivatives, then the scalar invariants calculated by the Riemann tensor are finite and it is safe to say that no singularity develops in the spacetime. While we perform the numerical calculation, we follow the evolution of the metric. Figure 3 shows logarithmic values of the metric terms $B, C$, and $H$ at several moments of time. They are smooth functions of $r$ and change also smoothly in $t$. We also calculate the Kretschmann scalar $R_{\alpha \beta \gamma \delta} R^{\alpha \beta \gamma \delta}$. This invariant is finite everywhere and every moment in time as shown in Fig. 4. Therefore, we conclude that the time-dependent treatment of supermassive strings makes a singularity-free spacetime possible. This treament is also useful to deal with the singularity of the global string in the next section.

As inflation proceeds at the core of the string, the metric terms $B, C$, and $H$ grow rapidly and the de Sitter expansion is established around the center of the string

$$
\frac{\dot{B}}{B} \approx \frac{\dot{C}}{C} \approx \frac{\dot{H}}{H} \approx \sqrt{\frac{8 \pi G}{3} V(\phi=0)} .
$$

In addition to radial inflation, the string also inflates along the axial direction at the core. As shown in Fig. 5, at the core, the ratio $\frac{\dot{H}}{H} / \frac{\dot{B}}{B}$ remains close to a constant $(\approx 1)$. This 
indicates that the expanding behavior along the $z$ - direction is similar to that along the $r-$ direction.

\section{GLOBAL STRING}

In this section, we consider the model of a $U(1)$ global string. The action, field equations, and the metric are the same as those of the gauge string in the previous section without the gauge field $\left(A_{\mu}=0\right)$. In the absence of the gauge field the scalar field configuration of a global string stretches further radially. The situation is equivalent to that of the gauge string with $\beta \rightarrow \infty(e \rightarrow 0)$. On the basis of the cause of inflation discussed in the previous section, this guarantees that the global string will inflate at a lower symmetry-breaking scale and that the inflationary picture should not be much different. For $n=1$, the critical value of $\eta$ for inflation is found to be $\eta_{c} \approx 0.23 m_{p}$ which is slightly lower than that of the gauge string. The critical value decreases as the winding number increases. The critical values are given for several winding numbers in Table $\llbracket$.

As mentioned in the Introduction, a static global string has a physical singularity outside the core. In this region, the energy-momentum tensor is given by

$$
T_{0}^{0}=T_{1}^{1}=T_{3}^{3}=-T_{2}^{2}=\frac{\eta^{2}}{g_{22}}
$$

and the closed-form solution of the Einstein equations was found by Cohen and Kaplan 9]

$$
d s^{2}=\left(\frac{u}{u_{0}}\right)\left(-d t^{2}+d z^{2}\right)+\gamma^{2}\left(\frac{u_{0}}{u}\right)^{1 / 2} e^{\frac{u_{0}^{2}-u^{2}}{u_{0}}}\left(d u^{2}+d \theta^{2}\right),
$$

where $u_{0}=1 / 8 \pi G \eta^{2}$, and $\gamma$ is a constant of integration. This metric is transformed to the linearized solution obtained by Harari and Sikivie [10] when $\eta \ll m_{p}$,

$$
d s^{2}=[1-4 G \mu(r)]\left(-d t^{2}+d z^{2}\right)+d r^{2}+[1-8 G \mu(r)] r^{2} d \theta^{2} .
$$

Here $\mu(r) \simeq \int_{\delta}^{r} T_{0}^{0} 2 \pi r d r \simeq 2 \pi \eta^{2} \ln (r / \delta)$ is the string mass per unit length out to a distance scale $r$ from the core $(\approx \delta)$. The relation between $u$ and $r$ is $u \approx u_{0}-\ln (r / \delta)$. Then the boundary of the core in the metric (3.2) is located at $u \simeq u_{0}$ and $u=\infty$ corresponds to the 
center of the string. The solution (3.2) exhibits coordinate singularities at $u=0$ and $u=\infty$. The one at $u=0$ turns out to be a physical one and the existence of this singularity was also examined by analyzing the field equations in Refs. [11,12]. For Grand-Unified-Theory scale strings $\left(\eta \sim 10^{16} \mathrm{GeV} \sim 10^{-3} m_{p}\right)$, the singularity is expelled outside the horizon, therefore, it does not cause any cosmological trouble. However, for supermassive strings, it is located near the core and could thus be problematic.

In a similar way to the supermassive gauge string, one way to escape from this trouble is to introduce a time-dependent string. Indeed, Gregory [13] removed the singularity by taking a specific time-dependent form of the metric: only axial time dependence was introduced. But this metric still has a coordinate singularity (not a physical one). It was later questioned by Wang and Nogales [17] that this coordinate singularity is unstable to both test particles and physical perturbations. In particular, they argued that the back reaction of the perturbations of null dust fluids will turn the coordinate singularity into a physical one. However, the situation is quite different for the supermassive strings. Because of the dynamical nature of the supermassive strings, we need to solve the system with a general time-dependent metric and scalar field.

We now solve the equations numerically as we did for the gauge string. Fig. 6 shows the logarithmic values of the metric terms $B, C$, and $H$ and Fig. 7 shows the Kretschmann scalar $R_{\alpha \beta \gamma \delta} R^{\alpha \beta \gamma \delta}$ at several moments of time. Their regular behaviors allow us to conclude that no singularity develops in the spacetime of a global string. For large $\eta$ global strings like supermassive gauge strings, the appropriate way to deal with their dynamical nature is to use a time-dependent treatment.

\section{CONCLUSIONS}

We have investigated inflation in cosmic strings. In the core region, the strings undergo inflation radially as well as axially when $\eta \gtrsim \eta_{c}$. With unit winding number $(n=1)$ the critical values for inflation were found to be $\eta_{c} \approx 0.25 m_{p}$ for a gauge string in the Bogomol'nyi 
limit $(\beta=1)$ and $\eta_{c}=0.23 m_{p}$ for a global string. The critical values decrease as $n$ and $\beta$ increase. We have explained this $\eta_{c}$ variation in terms of the core size of defects. The core of defects inflates when its size becomes bigger than the horizon scale: for larger $n$ and $\beta$, strings have bigger cores, and the global string has a bigger core than the gauge string for a given $\eta$. Regardless of the symmetry-breaking scale $\eta$, around the center of defects the de Sitter expansion is established since the scalar field stays about the top of the potenital $(\phi \approx 0)$. However, this is not sufficient for the cores of defects to inflate. Inflation requires another condition which is the core size being comparable to the horizon scale so that the core can be dynamical due to the gravitational effect. Or equivalently, the potential $V(\phi)$ needs to be flat enough at $\phi \approx 0$ so that the field $\phi$ can spend enough time about the top of the potential. For this condition to be satisfied, the symmetry-breaking scale $\eta$ needs to be sufficiently large. This description also explains why we have somewhat lower critical values of $\eta$ for strings than those for domain walls and global monopoles $\left(\eta_{c} \approx 0.33 m_{p}\right)$. Strings have bigger cores at the same symmetry-breaking scale than the other defects.

For supermassive gauge strings and all scale global strings, we have had troublesome physical singularities outside the core when we treat them in a static way. The elegant exit to nonsingular spacetimes is to introduce a time-dependent treatment. From the numerical simulations we could show that there is no singularity developing around time-dependent supermassive strings.

\section{ACKNOWLEDGMENTS}

Author is grateful to Alex Vilenkin, Pablo Laguna, Tanmay Vachaspati, and Xavier Siemens for helpful discussions and to National Science Foundation for partial support.

\section{APPENDIX: FIELD EQUATIONS AND NUMERICAL ALGORITHMS}

Einstein's equations with the metric (2.3) and the energy-momentum tensor (2.5) are 


$$
\begin{aligned}
& -G_{0}^{0}=K_{1}^{1} K_{2}^{2}+K_{2}^{2} K_{3}^{3}+K_{3}^{3} K_{1}^{1} \\
& +\frac{1}{B^{2}}\left(-\frac{C^{\prime \prime}}{C}-\frac{H^{\prime \prime}}{H}+\frac{B^{\prime}}{B} \frac{C^{\prime}}{C}-\frac{C^{\prime}}{C} \frac{H^{\prime}}{H}+\frac{H^{\prime}}{H} \frac{B^{\prime}}{B}+\frac{B^{\prime}}{B r}-2 \frac{C^{\prime}}{C r}-\frac{H^{\prime}}{H r}\right) \\
& =8 \pi G\left[\dot{\phi}^{2}+\frac{\phi^{\prime 2}}{B^{2}}+\frac{n^{2}}{C^{2} r^{2}} \phi^{2}(1-\alpha)^{2}+\frac{n^{2}}{2 e^{2}} \frac{1}{C^{2} r^{2}}\left(\dot{\alpha}^{2}+\frac{\alpha^{2}}{B^{2}}\right)+V(\phi)\right] \text {, } \\
& \frac{1}{2}\left(-G_{1}^{1}+G_{2}^{2}+G_{3}^{3}\right)=\dot{K}_{1}^{1}-\left(K_{1}^{1}\right)^{2}-\frac{1}{2}\left(K_{1}^{1} K_{2}^{2}-K_{2}^{2} K_{3}^{3}+K_{3}^{3} K_{1}^{1}\right) \\
& +\frac{1}{2 B^{2}}\left(\frac{C^{\prime \prime}}{C}+\frac{H^{\prime \prime}}{H}-\frac{B^{\prime}}{B} \frac{C^{\prime}}{C}-\frac{C^{\prime}}{C} \frac{H^{\prime}}{H}-\frac{H^{\prime}}{H} \frac{B^{\prime}}{B}-\frac{B^{\prime}}{B r}+2 \frac{C^{\prime}}{C r}-\frac{H^{\prime}}{H r}\right) \\
& =4 \pi G\left[\dot{\phi}^{2}-3 \frac{\phi^{\prime 2}}{B^{2}}+\frac{n^{2}}{C^{2} r^{2}} \phi^{2}(1-\alpha)^{2}-\frac{n^{2}}{2 e^{2}} \frac{1}{C^{2} r^{2}}\left(\dot{\alpha}^{2}+\frac{\alpha^{2}}{B^{2}}\right)-V(\phi)\right] \text {, } \\
& \frac{1}{2}\left(G_{1}^{1}-G_{2}^{2}+G_{3}^{3}\right)=\dot{K}_{2}^{2}-\left(K_{2}^{2}\right)^{2}-\frac{1}{2}\left(K_{1}^{1} K_{2}^{2}+K_{2}^{2} K_{3}^{3}-K_{3}^{3} K_{1}^{1}\right) \\
& +\frac{1}{2 B^{2}}\left(\frac{C^{\prime \prime}}{C}-\frac{H^{\prime \prime}}{H}-\frac{B^{\prime}}{B} \frac{C^{\prime}}{C}+\frac{C^{\prime}}{C} \frac{H^{\prime}}{H}+\frac{H^{\prime}}{H} \frac{B^{\prime}}{B}-\frac{B^{\prime}}{B r}+2 \frac{C^{\prime}}{C r}+\frac{H^{\prime}}{H r}\right) \\
& =4 \pi G\left[\dot{\phi}^{2}+\frac{\phi^{\prime 2}}{B^{2}}-3 \frac{n^{2}}{C^{2} r^{2}} \phi^{2}(1-\alpha)^{2}+\frac{n^{2}}{2 e^{2}} \frac{1}{C^{2} r^{2}}\left(3 \dot{\alpha}^{2}-\frac{\alpha^{\prime 2}}{B^{2}}\right)-V(\phi)\right] \text {, } \\
& \frac{1}{2}\left(G_{1}^{1}+G_{2}^{2}-G_{3}^{3}\right)=\dot{K}_{3}^{3}-\left(K_{3}^{3}\right)^{2}-\frac{1}{2}\left(-K_{1}^{1} K_{2}^{2}+K_{2}^{2} K_{3}^{3}+K_{3}^{3} K_{1}^{1}\right) \\
& +\frac{1}{2 B^{2}}\left(-\frac{C^{\prime \prime}}{C}+\frac{H^{\prime \prime}}{H}+\frac{B^{\prime}}{B} \frac{C^{\prime}}{C}+\frac{C^{\prime}}{C} \frac{H^{\prime}}{H}-\frac{H^{\prime}}{H} \frac{B^{\prime}}{B}+\frac{B^{\prime}}{B r}-2 \frac{C^{\prime}}{C r}+\frac{H^{\prime}}{H r}\right) \\
& =4 \pi G\left[\dot{\phi}^{2}+\frac{\phi^{\prime 2}}{B^{2}}+\frac{n^{2}}{C^{2} r^{2}} \phi^{2}(1-\alpha)^{2}-\frac{n^{2}}{2 e^{2}} \frac{1}{C^{2} r^{2}}\left(\dot{\alpha}^{2}-3 \frac{\alpha^{2}}{B^{2}}\right)-V(\phi)\right] \text {, } \\
& G_{01}=K_{2}^{2 \prime}+K_{3}^{3 \prime}-\left(K_{1}^{1}-K_{2}^{2}\right)\left(\frac{C^{\prime}}{C}+\frac{1}{r}\right)-\left(K_{1}^{1}-K_{3}^{3}\right) \frac{H^{\prime}}{H} \\
& =2 \dot{\phi} \phi^{\prime}+\frac{n^{2}}{e^{2}} \frac{1}{C^{2} r^{2}} \dot{\alpha} \alpha^{\prime}
\end{aligned}
$$

where

$$
K_{1}^{1}=-\frac{\dot{B}}{B}, \quad K_{2}^{2}=-\frac{\dot{C}}{C}, \quad K_{3}^{3}=-\frac{\dot{H}}{H}
$$

The field equation for $\phi$ is

$\ddot{\phi}-\left(K_{1}^{1}+K_{2}^{2}+K_{3}^{3}\right) \dot{\phi}-\frac{\phi^{\prime \prime}}{B^{2}}+\frac{1}{B^{2}}\left(\frac{B^{\prime}}{B}-\frac{C^{\prime}}{C}-\frac{H^{\prime}}{H}-\frac{1}{r}\right) \phi^{\prime}+\frac{n^{2}}{C^{2} r^{2}} \phi(1-\alpha)^{2}+\frac{\lambda}{2} \phi\left(\phi^{2}-\eta^{2}\right)=0$. 
The field equation for $\alpha$ is

$$
\ddot{\alpha}-\left(K_{1}^{1}-K_{2}^{2}+K_{3}^{3}\right) \dot{\alpha}-\frac{\alpha^{\prime \prime}}{B^{2}}+\frac{1}{B^{2}}\left(\frac{B^{\prime}}{B}+\frac{C^{\prime}}{C}-\frac{H^{\prime}}{H}+\frac{1}{r}\right) \alpha^{\prime}+2 e^{2} \phi^{2}(\alpha-1)=0 .
$$

The field equations for the global string are obtained by setting $\alpha=0$. At $t=0$, we assume a flat spacetime, $B(0, r)=C(0, r)=H(0, r)=1$, and zero velocities of scalar and gauge fields, $\dot{\phi}(0, r)=\dot{\alpha}(0, r)=0$. Then we solve the scalar and vector field equations (A7) and (A8) to obtain $\phi(0, r)$ and $\alpha(0, r) . K_{1}^{1}(0, r)$ and $K_{2}^{2}(0, r)$ are evaluated by the Hamiltonian and momentum constraint equations (A1) and (A5) after setting $K_{3}^{3}(0, r)=0$. In the next time step, $B(t, r), C(t, r)$, and $H(t, r)$ are calculated by Eq. (A6). The $K_{i}^{i}(t, r)$ 's are calculated by Eqs. (A2)-(A4), and $\phi(t, r)$ and $\alpha(t, r)$ are calculated by Eqs. (A7) and (A8). The boundary conditions for $\phi$ and $\alpha$ are given in Eq. (2.4). We also use the boundary condition $H^{\prime}(t, 0)=0\left(K_{3}^{3 \prime}=0\right)$ and apply regularity condition $K_{1}^{1}(t, 0)=K_{2}^{2}(t, 0)$ at the origin. To solve the field equations numerically, we use the modified Crank-Nicholson method. We insist that the Hamiltonian and momentum constraint equations, (A1) and (A5), are satisfied at every moment although they are not solved directly.

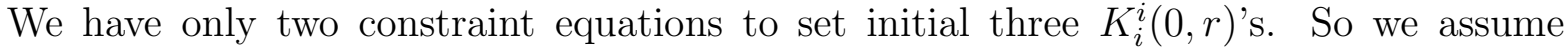
$K_{3}^{3}(0, r)=0$ which is consistent with the Einstein's equations. Nonetheless, it might not be the best choice. However, even if this choice is not the optimal one to describe our physical system, the numerical system will find its correct route propagating with the speed of light from the center of the string. The region in which we are interested relaxes to the correct configuration during the period of our numerical iteration. 


\section{REFERENCES}

[1] A. Vilenkin, Phys. Rev. Lett. 72, 3137 (1994).

[2] A. Linde, Phys. Lett. B327, 208 (1994).

[3] N. Sakai, H. Shinkai, T. Tachizawa, and K. Maeda, Phys. Rev. D53, 655 (1996).

[4] A. de Laix, M. Trodden and T. Vachaspati, preprint gr-qc/9801016.

[5] A. Vilenkin, Phys. Rev. D23, 852 (1981).

[6] J. Gott, Ap. J. 288, 422 (1985).

[7] M. Ortiz, Phys. Rev. D43, 2521 (1991).

[8] P. Laguna and D. Garfinkle, Phys. Rev. D40, 1101 (1989).

[9] A. Cohen and D. Kaplan, Phys. Lett. B215, 67 (1988).

[10] D. Harari and P. Sikivie, Phys. Rev. D37, 3438 (1988).

[11] R. Gregory, Phys. Lett. B215, 663 (1988).

[12] G. Gibbons, M. Ortiz and F. Ruiz, Phys. Rev. D39, 1546 (1989).

[13] R. Gregory, Phys. Rev. D54, 4955 (1996).

[14] B. Linet, Phys. Lett. A124, 240 (1987).

[15] T. Levi-Civita, Atti Acc. Naz. Lincei. Rend. 28, 101 (1919).

[16] E. Kasner, Am. J. Math. 43, 217 (1921).

[17] A. Wang and J. Nogales, Phys. Rev. D56, 6217 (1997). 


\section{TABLES}

\begin{tabular}{ccccc}
\hline \hline $\mathrm{n}$ & 1 & 2 & 3 & 4 \\
\hline$\eta_{c} / m_{p}$ & $0.25 \pm 0.005$ & $0.17 \pm 0.005$ & $0.13 \pm 0.005$ & $0.11 \pm 0.005$ \\
\hline \hline
\end{tabular}

(a)

\begin{tabular}{cccccc}
\hline \hline$\beta$ & 0.5 & 1 & 2 & 3 & 4 \\
\hline$\eta_{c} / m_{p}$ & $0.255 \pm 0.0025$ & $0.25 \pm 0.0025$ & $0.24 \pm 0.0025$ & $0.235 \pm 0.0025$ & $0.235 \pm 0.0025$ \\
\hline \hline
\end{tabular}

(b)

TABLE I. (a) The critical values of $\eta$ for the gauge string in the Bogomol'nyi limit $\beta=1$. (b) The critical values of $\eta$ for $n=1$.

\begin{tabular}{cccccc}
\hline \hline $\mathrm{n}$ & 1 & 2 & 3 & 4 & 5 \\
\hline$\eta_{c} / m_{p}$ & $0.23 \pm 0.0025$ & $0.155 \pm 0.0025$ & $0.115 \pm 0.0025$ & $0.095 \pm 0.0025$ & $0.075 \pm 0.0025$ \\
\hline \hline
\end{tabular}

TABLE II. The critical values of $\eta$ for the global string 


\section{FIGURES}

FIG. 1. A plot of $\delta / H_{0}^{-1}$ vs. $n$ for the $\eta=0.1,0.5,1.0,2.0,3.0 m_{p}$ gauge strings (from the bottom to the top) in the flat spacetime. The dashed line corresponds to $\sqrt{n}$ as a reference. $H_{0}^{-1}$ is the horizon size at the center of the string.

FIG. 2. The scalar field configurations as functions of the proper radius $C H_{0} r$ at $H_{0} t=0,2,4$ (from the left to the right) for the $\eta=0.5 m_{p}$ gauge string $(n=1, \beta=1)$. The rapid growth of the proper radius in the core region indicates inflation.

FIG. 3. Plots of (a) $\log _{10} B, \log _{10} C$, and (b) $\log _{10} H$ vs. $H_{0} r$ at $H_{0} t=1,3,5$ (from the bottom to the top) for the $\eta=0.5 m_{p}$ gauge string $(n=1, \beta=1)$. The metric terms behave regularly in $r$ and $t$.

FIG. 4. A plot of $R_{\alpha \beta \gamma \delta} R^{\alpha \beta \gamma \delta} / H_{0}^{4}$ vs. $H_{0} r$ at $H_{0} t=2,4,6$ (from the right to the left) for the $\eta=0.5 m_{p}$ gauge string $(n=1, \beta=1)$. The scalar invariant is finite everywhere. The generic picture is not very different for different $\eta$ 's.

FIG. 5. A plot of $\frac{\dot{H}}{H} / \frac{\dot{B}}{B}$ vs. $H_{0} r$ at $H_{0} t=2,3,4,5$ (from the bottom to the top) for the $\eta=0.5 m_{p}$ gauge string $(n=1, \beta=1)$. The ratio stays close to a constant $(\approx 1)$ in the core region $\left(H_{0} r \lesssim 0.5\right)$.

FIG. 6. Plots of (a) $\log _{10} B, \log _{10} C$, and (b) $\log _{10} H$ vs. $H_{0} r$ at $H_{0} t=1,3,5$ (from the bottom to the top) for the $\eta=0.4 m_{p}$ global string $(n=1)$. The metric terms behave regularly in $r$ and $t$.

FIG. 7. A plot of $R_{\alpha \beta \gamma \delta} R^{\alpha \beta \gamma \delta} / H_{0}^{4}$ vs. $H_{0} r$ at $H_{0} t=2,4,6$ (from the right to the left) for the $\eta=0.4 m_{p}$ global string $(n=1)$. The scalar invariant is finite everywhere. The generic picture is not very different for different $\eta$ 's. The core is located at $H_{0} r \lesssim 0.5$. 


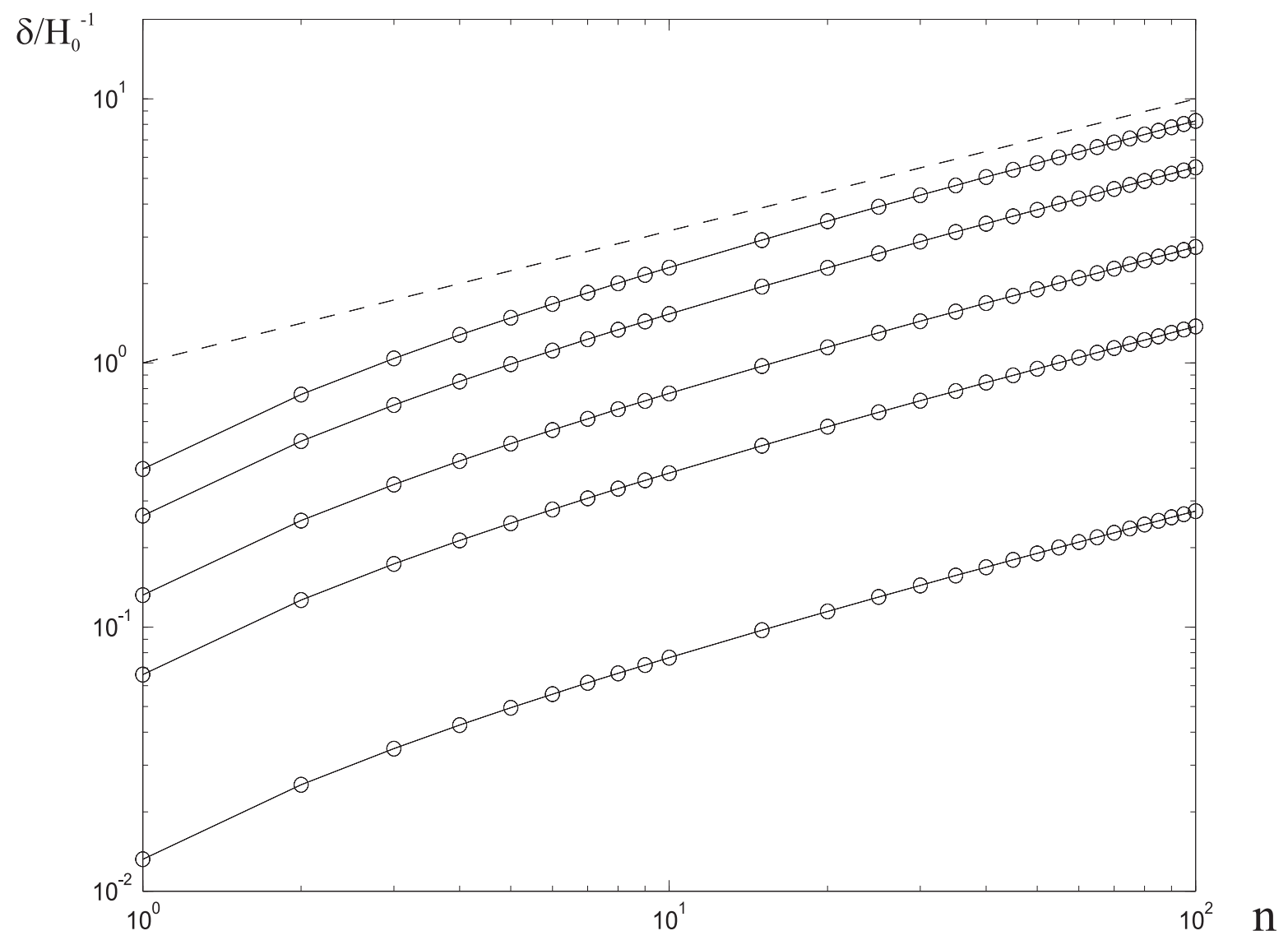


$\phi / \eta$

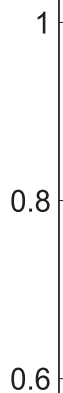

0.6

0.4

0.2

0

5

10

15

20

25

30

$35 \mathrm{Cr} / \mathrm{H}_{0}^{-1}$ 


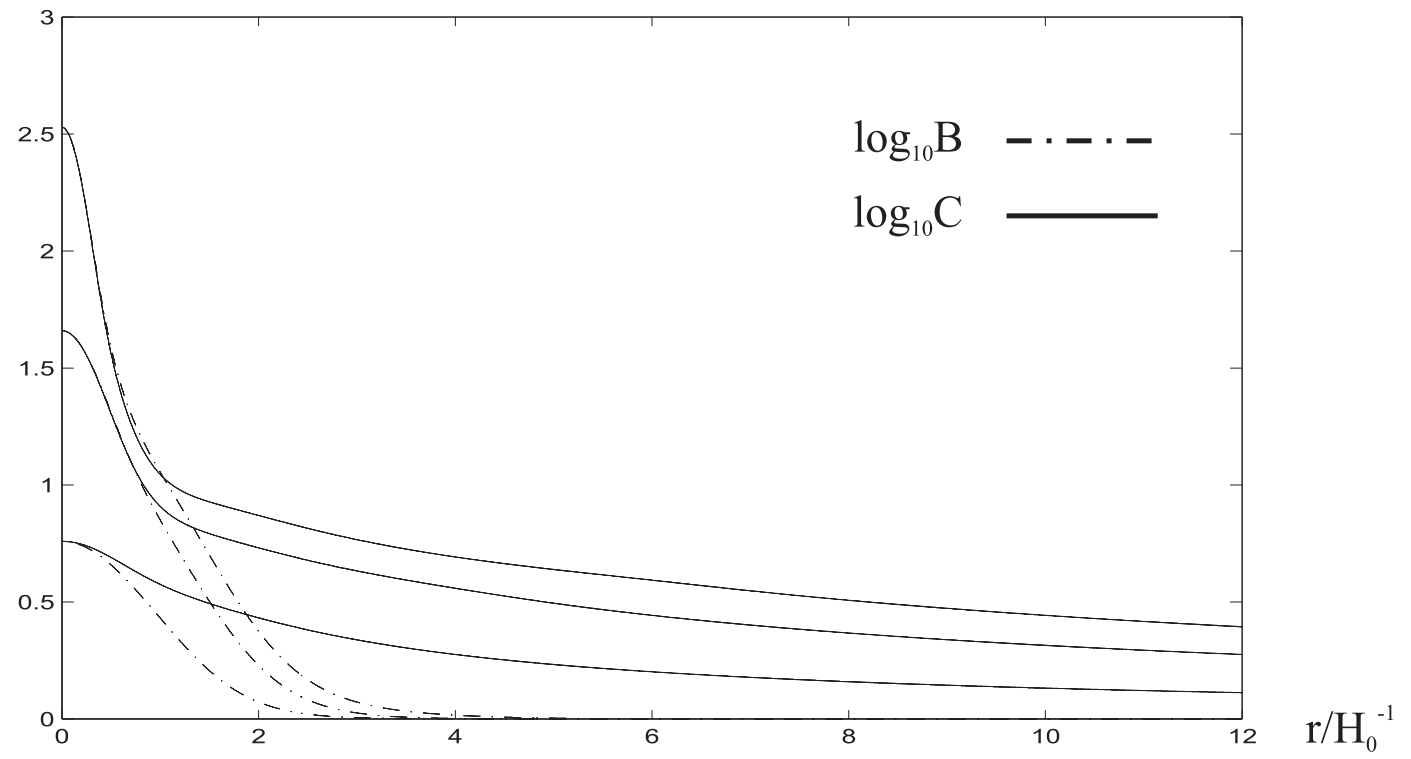

(a)

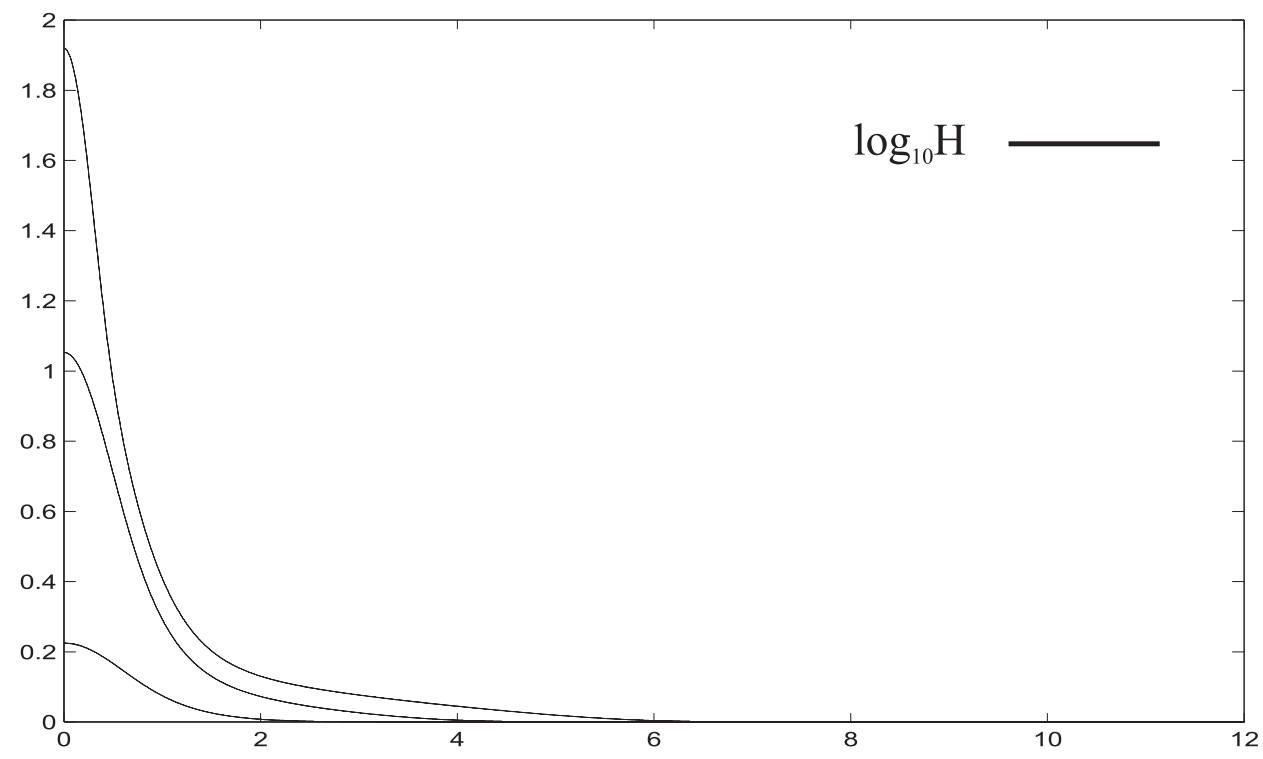

(b) 


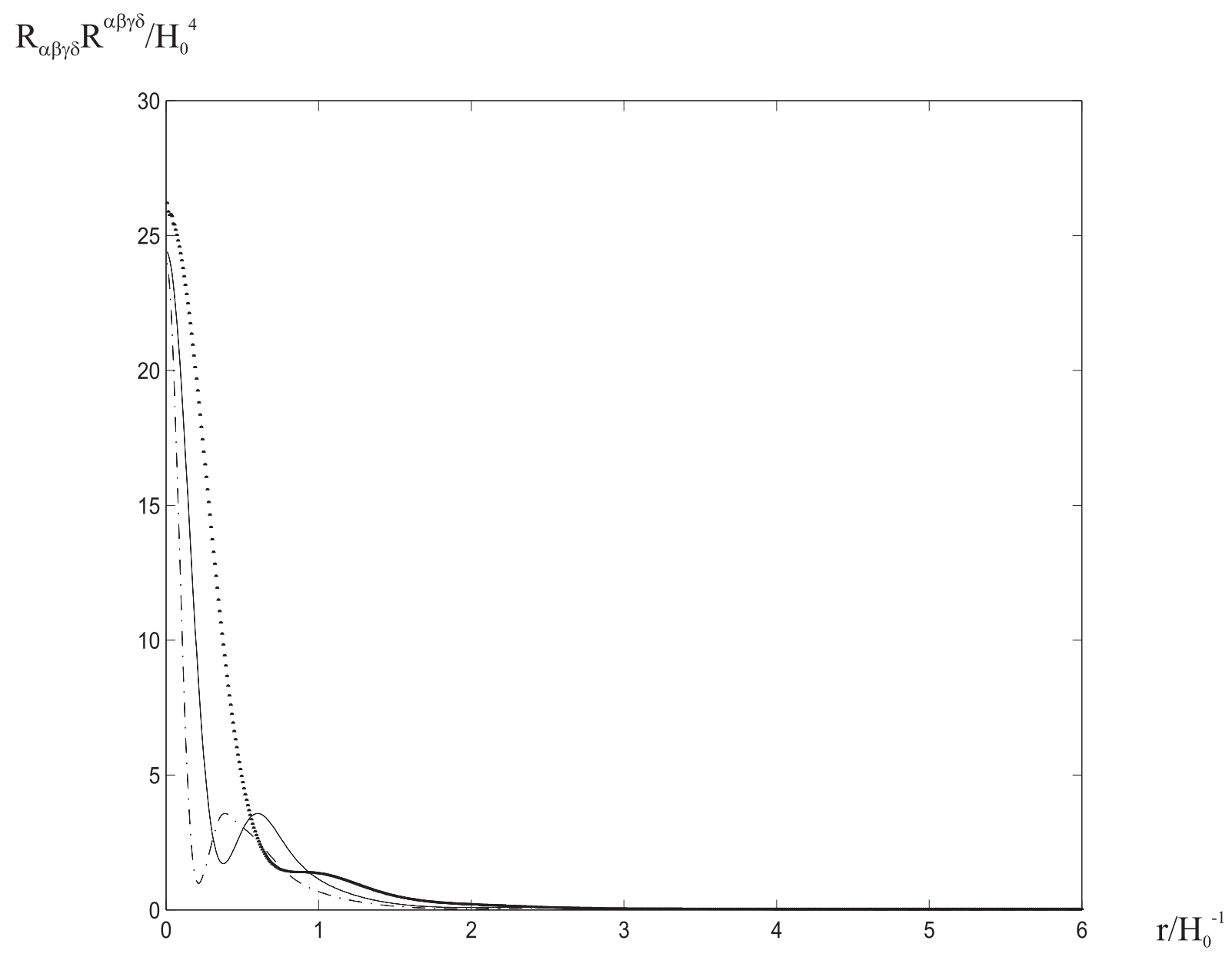




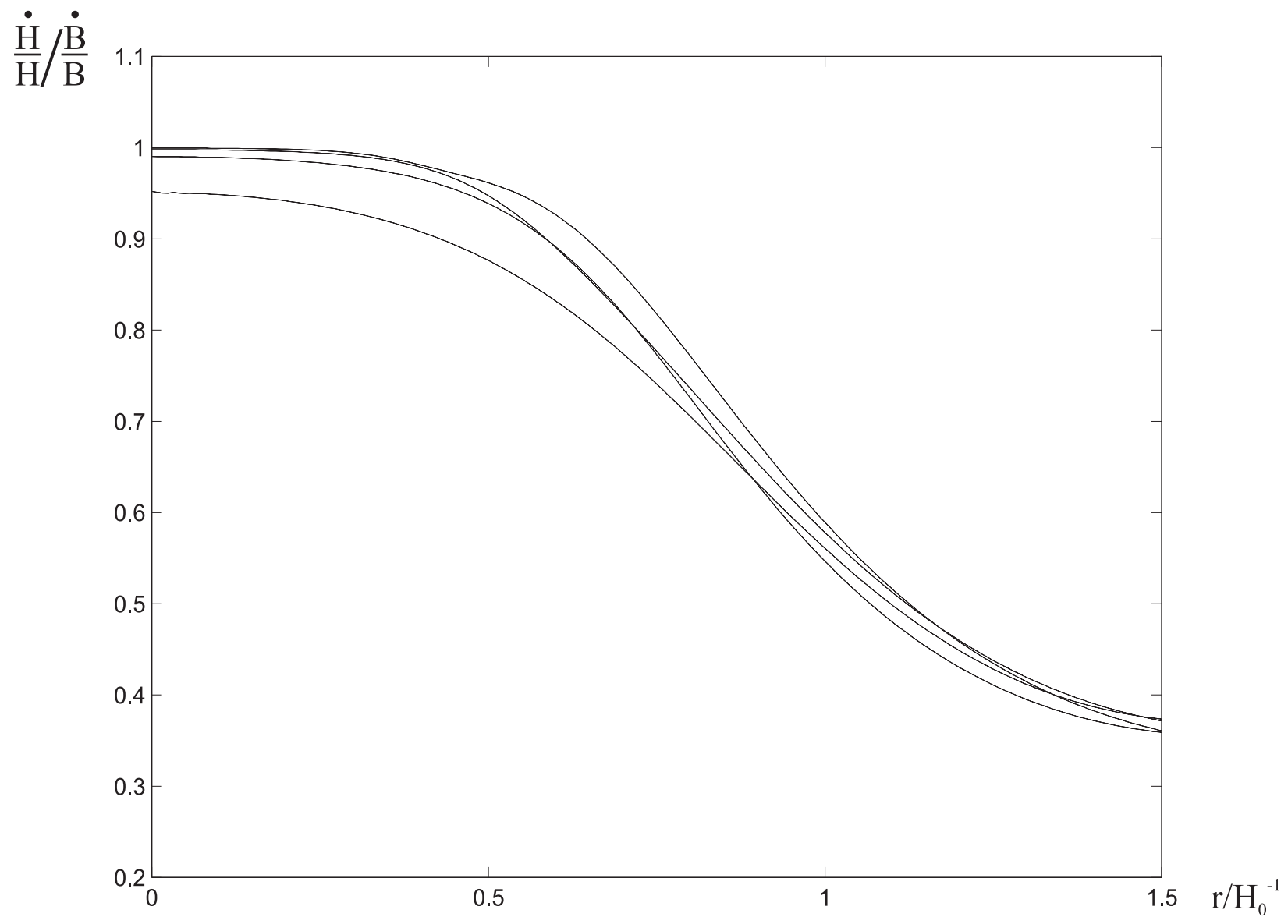




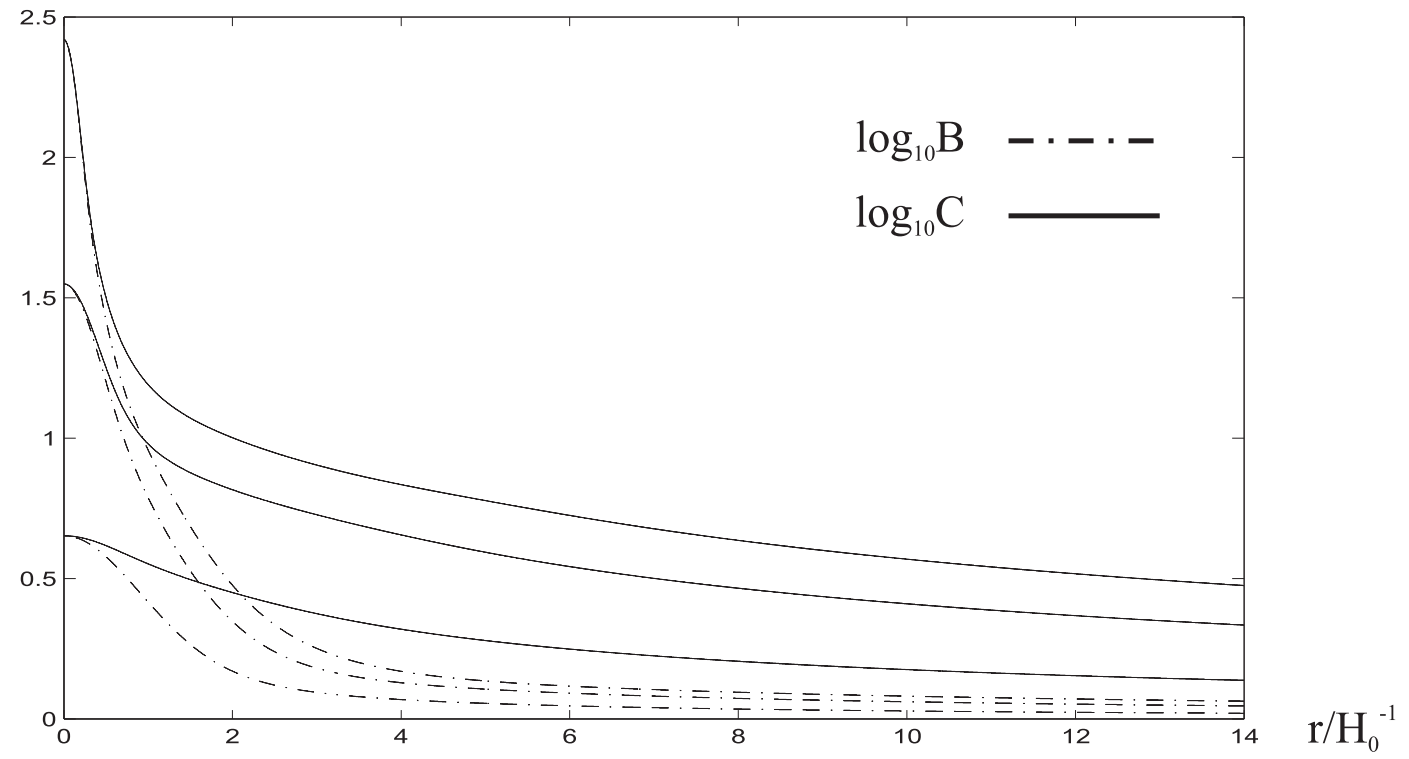

(a)

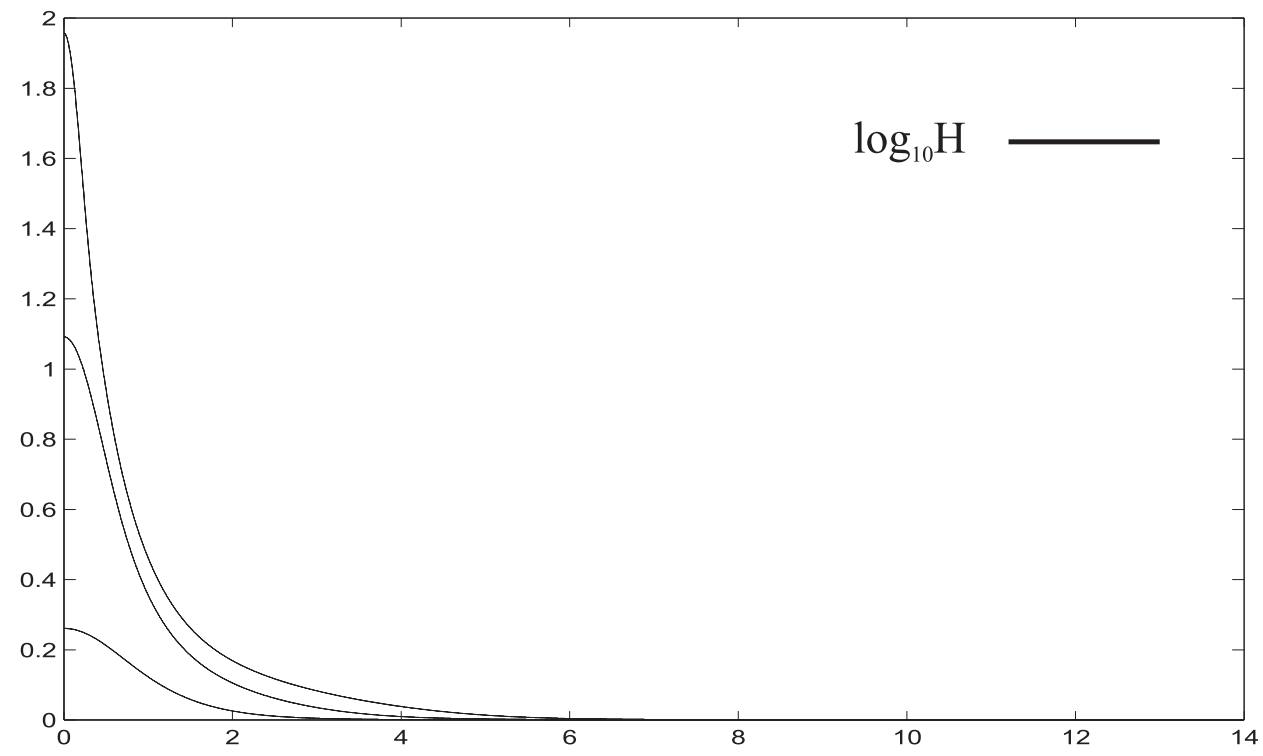

(b) 


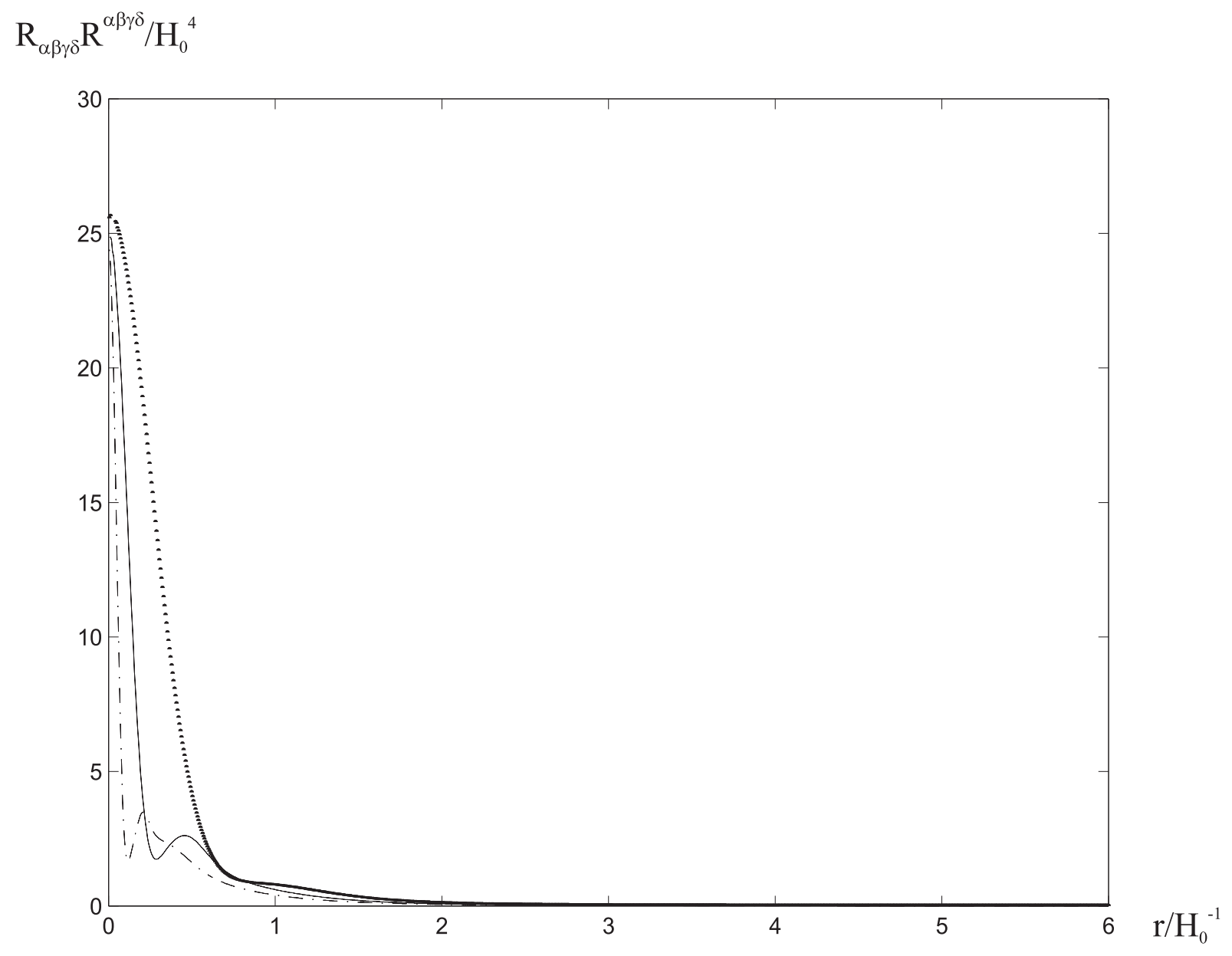

